the site of the block more accurately and so to increase our understanding of the conventional, surface electrocardiogram, ${ }^{3}$ but use of the surface electrocardiogram alone to distinguish nodal from His-Purkinje disease remains difficult and may occasionally be misleading. Nevertheless, some guidance ${ }^{1}$ may be given by the width of the QRS complex (narrow complexes suggesting that the block is nodal) and the response of the heart rate to atropine and to exercise (failure of response to either of these manoeuvres suggesting His-Purkinje disease). If the heart rate is over 50 per minute that, too, may indicate that the block is located in the atrioventricular node.

The use of these variables to distinguish dangerous from benign complete heart block has recently been questioned. ${ }^{4}$ In the 29 patients studied by Lane and Kennelly ${ }^{4}$ the resting heart rate did not distinguish symptomatic from asymptomatic patients with either wide or narrow QRS complexes. Intracardiac recordings of the site of the block were also unhelpful. They claimed that the best test for identifying patients at risk of serious symptoms was the response of the heart to right ventricular pacing. In technical terms, the mathematical product of the ventricular escape time and the length of the overdrive cycle seemed to have a predictive value. Nevertheless, while there were statistically significant differences between groups the overlap was too great for this test alone to be of clinical value in individual patients.

For the time being, therefore, the physician wanting guidance on whether or not to advise prophylactic pacing for his patients will have to continue to use his clinical judgment. One group of patients who may not require permanent pacing are those whose block is localised to the atrioventricular node and in whom the heart rate accelerates with atropine or exercise. Failure of the ventricular rate to be suppressed by right ventricular pacing strengthens the argument. Nevertheless, whenever a decision is made not to advise insertion of a pacemaker the patient's progress must be followed carefully.

In patients in whom treatment is considered justifiable the best treatment is implantation of a permanent pacing system using either an endocardial or epicardial electrode. Should pacing not be possible, patients whose heart rate is increased by intravenous isoprenaline ${ }^{5}$ may benefit from treatment with a long-acting preparation of the drug. ${ }^{6}$

${ }^{1}$ Narula, O S, Circulation, 1970, 41, 437.

${ }^{2}$ Langendorf, R, and Pick, A, Circulation, 1968, 38, 819.

${ }^{3}$ Puech, P, in Cardiac Arhythmias. The Modern Electrophysiological Approach, ed D M Krikler and J F Goodwin, p 81. London, Saunders, 1975.

${ }^{4}$ Lane, G K, and Kennelly, B M, Cardiovascular Research, 1978, 12, 712.

${ }^{5}$ Redwood, D, British Medical fournal, 1968, 1, 419.

${ }^{6}$ Fleming, H A, and Bailey, S M, British Heart fournal, 1972, 34, 309.

\section{Drug toxicity studies and the clinical pharmacologist}

We have no reliable estimate of the number of hospital admissions in Britain each year attributable to drug reactions. One report ${ }^{1}$ suggested that about $3.5 \%$ of medical admissions are of this type, with a further $2.5 \%$ attributable to deliberate drug overdosage ${ }^{1}$ (relatively few patients being admitted to wards other than medical-or paediatric-for these reasons ${ }^{2}$ ). Most drug reactions are mild, but a few may be severe enough to threaten life-a possibility more likely in patients with serious or terminal illness. ${ }^{3}$ Nevertheless, in such circumstances these risks may seem worth taking when balanced against possible benefits.

Early in the 1960s Wade and his colleagues in Northern Ireland showed how variable was the prescription of oral hypoglycaemic drugs in the province. ${ }^{4}$ Such variability has since been confirmed for many other drugs elsewhere. It stems as much from differences between doctors as from fluctuations in the local prevalence of disease. More recently McDevitt and his colleagues ${ }^{5}$ and the Liverpool Therapeutics Group ${ }^{6}$ have shown that many patients in domiciliary practice who take digoxin regularly do equally well without it. Yet here is a drug with a substantial risk of undesired effects. No doubt before long some patients with cardiac failure will be reported not to need long-term diuretic treatment once the initial episode has been treated adequately. Similarly, at least in the United States, most maturity-onset diabetic patients who adhere to their diets have been shown not to need oral hypoglycaemic agents.

Clearly we need more studies of drug-prescribing habits, aimed particularly at discovering the reasons why some doctors are enthusiastic and others conservative drug prescribers. Also needed are more studies into long-term use of potentially toxic drugs such as steroids (both oral and topical), anticoagulants, and diuretics. Despite much interest in the last few decades there have been few systematic studies on the frequency of adverse drug reactions in defined populations. The Boston Collaborative Drug Surveillance Program has a wealth of information and continues to publish regular reports. Much simpler studies can, however, be undertaken for a more limited number of drugs. ${ }^{78}$ Unfortunately, few groups in Britain have undertaken this type of work, though the recent report in the $B M F$ of acute toxicity from frusemide in patients in hospital is an example of the type of study we urgently need. ${ }^{9}$ The problems of quantification of risk are much greater in outpatients. Here Britain can take justified pride in the pioneering work done by Skegg and Doll, ${ }^{10}$ with their study of over 40000 patients in several general practices in the Oxford area.

In the future this type of study must, surely, become the province of the clinical pharmacologist. Professor M Rawlins of Newcastle has recently suggested that there is a case for devolution of some of the functions of the Adverse Reaction Subcommittee of the Committee on Safety of Medicines, which is based at present in London. At least part of this work could be undertaken more readily by regional clinical pharmacology centres with their improved contacts with local prescribers. Such centres could collaborate in collating spontaneous reports of adverse effects from prescribers and share responsibility for conducting studies on acute drug toxicity. This could be done at minimal cost and would rapidly increase our knowledge of the subgroups of the population at high risk of reasonably common serious adverse drug effects. The experience gained could then be put to immediate practical use since it would help in developing the postmarketing surveillance studies we so urgently need for new products.

${ }^{1}$ Miller, R R, Archives of Internal Medicine, 1974, 134, 219.

${ }^{2}$ Hurwitz, N, British Medical fournal, 1969, 1, 539.

${ }^{3}$ Lawson, D H, Hutcheon, A W, and Jick, H, Scottish Medical fournal, 1979, 24, 127.

4 Wade, O L, and Hood, H, British fournal of Preventive and Social Medicine, 1972, 26, 205.

${ }^{5}$ Johnston, G D, and McDevitt, D G, Lancet, 1979, 1, 567.

6 Breckenridge, A, et al, British Medical fournal, 1978, 2, 673.

7 Koch-Weser, J, et al, Annals of Internal Medicine, 1970, 72, 857.

${ }^{8}$ Koch-Weser, J, et al, Archives of Internal Medicine, 1971, 128, 399.

${ }^{9}$ Lowe, J, et al, British Medical fournal, 1979, 2, 360.

10 Skegg, D C G, and Doll, R, Lancet, 1977, 2, 475. 